\title{
SOFT CONTROL OF SELF-ORGANIZED LOCALLY INTERACTING BROWNIAN PLANAR AGENTS
}

\author{
Guillaume Sartoretti^ and Max-Olivier Hongler \\ Polytechnical Federal School of Lausanne (EPFL) \\ CH-1015 Lausanne, Switzerland \\ \{guillaume.sartoretti, max.hongler\}@epfl.ch \\ http://www.epfl.ch
}

\begin{abstract}
This contribution is addressed to the dynamics of heterogeneous interacting agents evolving on the plane. Heterogeneity is due to the presence of an unfiltered externally controllable fellow, a shill, which via mutual interactions ultimately drives (i.e. soft controls) the whole society towards a given goal. We are able to calculate relevant dynamic characteristics of this controllable agent. This opens the possibility to optimize the soft controlling of a whole society by infiltrating it with a properly designed shill. Numerical results fully corroborate our theoretical findings.
\end{abstract}

Keywords: homogeneous and heterogeneous Brownian agents - limitedrange mutual interactions - soft control - mixed canonical-dissipative dynamics - mean-field description - analytical results.

\section{Introduction}

The Soft Control of a swarm of interacting agents consists in introducing an externally controllable agent into an homogeneous swarm of autonomous agents. By suitably chose the externally controllable fellow, one ultimately can (softly) control the whole swarm. While her dynamics differs, the controllable infiltrated agent, often referred to as the Shill ( $\mathrm{SH})$, is detected by the other fellows as being an ordinary member of the society. The SH's influence can be used to stimulate specific positive features to the whole group (optimal driving to targets, enhancement of flocking capability, extra energy scavenging, etc. [1-3]). Alternatively the SH's presence may destroy the overall swarm's coherence [1]. Soft control is also commonly used in ethology, by introducing robotic shills in societies of animals, see for instance [4-6].

In the sequel, we consider an homogeneous swarm of Brownian agents following a planar mixed canonical-dissipative dynamics as introduced in [7]. After showing how the agents asymptotically converge to (and circulate on) a self-selected closed annular orbits on $\mathbb{R}^{2}$, we then introduce a SH agent into the swarm. We then use the SH's presence to soft control the angular speed of the whole society.

\footnotetext{
* Supported by the Swiss National Funds for Scientific Research
} 


\section{Interacting Diffusion Processes on $\mathbb{R}^{2}$}

For $\overline{\mathbf{X}}(t)=\left(\mathbf{X}_{1}(t), \mathbf{X}_{2}(t), \cdots, \mathbf{X}_{N}(t)\right) \in \mathbb{R}^{2 N}$ with $\mathbf{X}_{k}(t)=\left(x_{1, k}(t), x_{2, k}(t)\right) \in$ $\mathbb{R}^{2}$, our class of dynamics is given by a collection of $N$ mutually interacting planar Brownian agents evolving according to a set of Stochastic Differential Equations (SDE) on $\mathbb{R}^{2 N}$ :

$$
\left\{\begin{array}{l}
d \mathbf{X}_{k}(t)=d t\left[\mathcal{A}_{k}(t)-\Lambda\left(\mathcal{L}_{k, \rho}(t)-\mathcal{H}\left(\mathbf{X}_{k}\right)\right)\right] \nabla \mathcal{H}\left(\mathbf{X}_{k}\right)+\sigma d \mathbf{W}_{k}(t), \\
\mathbf{X}_{k}(0)=\mathbf{X}_{0, k},
\end{array}\right.
$$

where $\sigma, \Lambda \in \mathbb{R}^{+}$are control parameters, and the definitions of the dynamical inputs are:

i) the Hamiltonian function $\mathcal{H}\left(x_{1}, x_{2}\right): \mathbb{R}^{2} \mapsto \mathbb{R}^{+}$are used to define a family of closed, non-intersecting, planar curves with equations given by

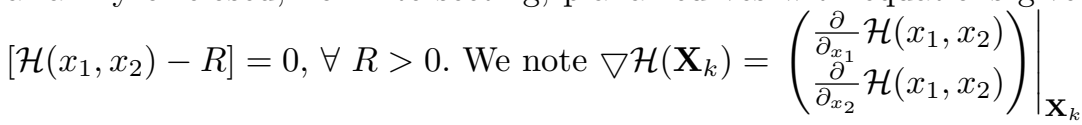

ii) the tangent dynamical map at the origin. In absence of noise source $(\sigma \equiv 0)$, the origin is a singular point of Eq.(1) and its associated linear map $\mathcal{A}_{k}(t)$ :

$$
\mathcal{A}_{k}(t)=\left(\begin{array}{cc}
\frac{N_{k, \rho}(t)}{N}-\frac{1}{M} & \frac{N_{k, \rho}(t)}{N} \\
-\frac{N_{k, \rho}(t)}{N} & \frac{N_{k, \rho}(t)}{N}-\frac{1}{M}
\end{array}\right)
$$

where $M \in[1, N] \subseteq \mathbb{R}^{+}$is a control parameter and $\left(N_{k, \rho}(t) / N\right)$ is the fraction of the total swarm detected by agent $a_{k}$ in the neighborhood $\mathcal{D}_{k, \rho}(t)$ defined as:

$$
\mathcal{D}_{k, \rho}(t)=\left\{\mathbf{X} \in \mathbb{R}^{2} \mid\left\|\mathbf{X}-\mathbf{X}_{k}(t)\right\|_{2} \leq \rho\right\}
$$

iii) the self-adaptive Hamiltonian level. The scalar quantity:

$$
\mathcal{L}_{k, \rho}(t):=\frac{1}{N_{k, \rho}(t)} \sum_{i \in V_{k, \rho}(t)} \mathcal{H}\left(\mathbf{X}_{i}\right)
$$

where $N_{k, \rho}(t)$ has been defined in ii). The summation extends over the agents' set $V_{k, \rho}(t):=\left\{i \mid \mathbf{X}_{i} \in \mathcal{D}_{k, \rho}(t)\right\}$

iv) the noise sources. $d \mathbf{W}_{k}(t)=\left(d W_{1, k}(t), d W_{2, k}(t)\right)$ are $2 N$ independent standard White Gaussian Noise (WGN) processes

Proposition 1. For $t \rightarrow \infty$, the dissipative dynamics Eq.(1) reaches a stationary regime characterized by the time-invariant probability product measure:

$P_{s}(\overline{\mathbf{x}}) d \mathbf{x}=\left[P_{s}(\mathbf{x}) d \mathbf{x}\right]^{N} \quad$ with $\quad P_{s}(\mathbf{x}) d \mathbf{x}=\mathcal{Z}^{-1} \exp \left\{-\frac{\Lambda}{\sigma^{2}}\left[\mathcal{L}_{s, \rho}-\mathcal{H}(\mathbf{x})\right]^{2}\right\} d \mathbf{x}$.

where $\mathcal{Z}$ is the normalization factor, and $\mathcal{L}_{s, \rho}$ is the stationary Hamiltonian level curve statistically reached by the agents. 


\section{Proof of Proposition 1.}

We first write the Fokker-Planck equation (FPE) associated with the diffusion process of Eq.(1):

$\partial_{t} P\left(\overline{\mathbf{x}}, t \mid \overline{\mathbf{x}}_{0}\right)=-\nabla \cdot\left\{\left[\mathcal{A}(t) \nabla \mathcal{H}(\mathbf{x})-\nabla V_{\rho}(\overline{\mathbf{x}}, t)\right] \cdot P\left(\overline{\mathbf{x}}, t \mid \overline{\mathbf{x}}_{0}\right)+\frac{\sigma^{2}}{2} \nabla P\left(\overline{\mathbf{x}}, t \mid \overline{\mathbf{x}}_{0}\right)\right\}$,

with $\mathcal{A}(t)$ the $(2 N \times 2 N)$ block-simplectic matrix

$$
\mathcal{A}(t):=\left(\begin{array}{ccc}
A_{1}(t) & & 0 \\
& \ddots & \\
0 & & A_{N}(t)
\end{array}\right), \quad \text { with } A_{k}(t)=\left(\begin{array}{cc}
0 & \frac{N_{k, \rho}(t)}{N} \\
-\frac{N_{k, \rho}(t)}{N} & 0
\end{array}\right)
$$

The dissipative component of the drift is derived from the time-dependent generalized potential $V_{\rho}(\overline{\mathbf{x}}, t)$, which reads as:

$$
V_{\rho}(\overline{\mathbf{x}}, t)=\sum_{k=1}^{N}\{\overbrace{\left[\frac{N_{k, \rho}(t)}{N}-\frac{1}{M}\right]}^{:=\mathcal{R}_{k, \rho}(t)}+\frac{\Lambda}{2}\left[\mathcal{L}_{k, \rho}(t)-\mathcal{H}\left(\mathbf{X}_{k}\right)\right]^{2}\} \nabla \mathcal{H}(\mathbf{x}) .
$$

The parameters $\mathcal{R}_{k, \rho}(t)$ and $\mathcal{L}_{k, \rho}(t)$ in Eq.(6) implicitly depend on the agents' configurations, implying that Eq.(5) is effectively a nonlinear FPE. The potential $V_{\rho}(\overline{\mathbf{x}}, t)$ is globally attractive on $\mathbb{R}^{2 N}$ (i.e $V_{\rho}(\overline{\mathbf{x}}, t) \rightarrow \infty$ for $\|\overline{\mathbf{x}}\| \rightarrow \infty$ ). Global attraction on $\mathbb{R}^{2 N}$ in Eq.(1) together with the WGN driving forces imply that the diffusive dynamics is ergodic. Hence, it exists an unique invariant measure $P_{s}(\overline{\mathbf{x}})$ implying that both $\mathcal{R}_{k, \rho}(t)$ and $\mathcal{L}_{k, \rho}(t)$ asymptotically converge towards stationary (time-independent) values $\mathcal{R}_{k, s, \rho}$ and $\mathcal{L}_{k, s, \rho}$. Moreover, as Eqs.(5) and (6) are invariant under permutations of the agents labeling, we shall have $\mathcal{R}_{k, s, \rho}=: \mathcal{R}_{s, \rho}$ and $\mathcal{L}_{k, s, \rho}=: \mathcal{L}_{s, \rho}(\forall k)$. Dissipation drives the system to its minimal energy configuration, leading to the specific values $\mathcal{R}_{s, \rho} \equiv 0$ and to $\lim _{t \rightarrow \infty} \mathcal{A}(t)=\mathcal{A}_{s}$ with:

$$
\mathcal{A}_{s}:=\left(\begin{array}{ccc}
A_{s} & & 0 \\
& \ddots & \\
0 & & A_{s}
\end{array}\right), \quad \mathcal{A}_{s}=\left(\begin{array}{cc}
0 & \frac{1}{M} \\
-\frac{1}{M} & 0
\end{array}\right) .
$$

Observe that the gradient $\nabla V_{\rho}(\overline{\mathbf{x}}, t)$ is systematically orthogonal to the antisymmetric component $\mathcal{A}(t) \nabla \mathcal{H}(\mathbf{x})$. This remains true in the stationary regime $\mathcal{A}_{s}$, allowing us to write the stationary solution of the FPE Eq.(5) in the product-form $P_{s}(\overline{\mathbf{x}})$ :

$$
\begin{aligned}
& P_{s}(\overline{\mathbf{x}})=\left[P_{s}(\mathbf{x})\right]^{N}=\left[\mathcal{N}^{-1} e^{\left\{V_{s, \rho}(\mathbf{x})\right\}}\right]^{N}, \\
& V_{s, \rho}(\mathbf{x})=+\frac{\gamma}{2}\left[\mathcal{L}_{s, \rho}-\mathcal{H}(\mathbf{x})\right]^{2}
\end{aligned}
$$

with $\overline{\mathbf{x}}=\left(\mathbf{x}_{1}, \mathbf{x}_{2}, \cdots, \mathbf{x}_{N}\right) \in \mathbb{R}^{2 N}, \mathbf{x}_{k} \in \mathbb{R}^{2}$. In Eq.(7), $\mathbf{x} \in \mathbb{R}^{2}$ stands for one representative agent in the swarm. The product form of $P_{s}(\overline{\mathbf{x}})$ explicitly shows that our dynamics propagates chaos, validating the use of a Mean Field (MF) approach, where the behavior of a single agent of the swarm effectively reflects the global dynamics.

Here the stationary regime of the single representative agent $\mathbf{X}(t)=\left(x_{1}(t), x_{2}(t)\right)$ is characterized by the invariant probability density $P_{s}(\mathbf{x})$ :

$$
0=-\nabla\left\{\left[\mathcal{A}_{s} \nabla \mathcal{H}(\mathbf{x})-\nabla V_{s, \rho}(\mathbf{x})\right] \cdot P_{s}(\mathbf{x})\right\}+\frac{\sigma^{2}}{2} \triangle P_{s}(\mathbf{x}),
$$


Auto-consistency of the nonlinear FPE here implies:

$$
\frac{N_{s, \rho}}{N}=\int_{\mathbf{x} \in D_{s, \rho}} P_{s}(\mathbf{x}) d x_{1} d x_{2}\left(=\frac{1}{M}\right)
$$

where here $D_{s, \rho}$ is the stationary representative agent's neighborhood.

Corollary 1. For the harmonic oscillator's Hamiltonian function

$$
\mathcal{H}\left(x_{1}, x_{2}\right)=1 / 2\left(x_{1}^{2}+x_{2}^{2}\right),
$$

and with $\Lambda \rightarrow \infty$, the stationary probability measure converges to a uniform distribution of the agents on the limit cycle. Following the argument in the caption of Figure 1, the radius of the limit cycle can be in this case explicitly written as

$$
\mathcal{L}_{s, \rho}=\frac{\rho}{\sqrt{2-2 \cos \left(\frac{\pi}{M}\right)}} .
$$
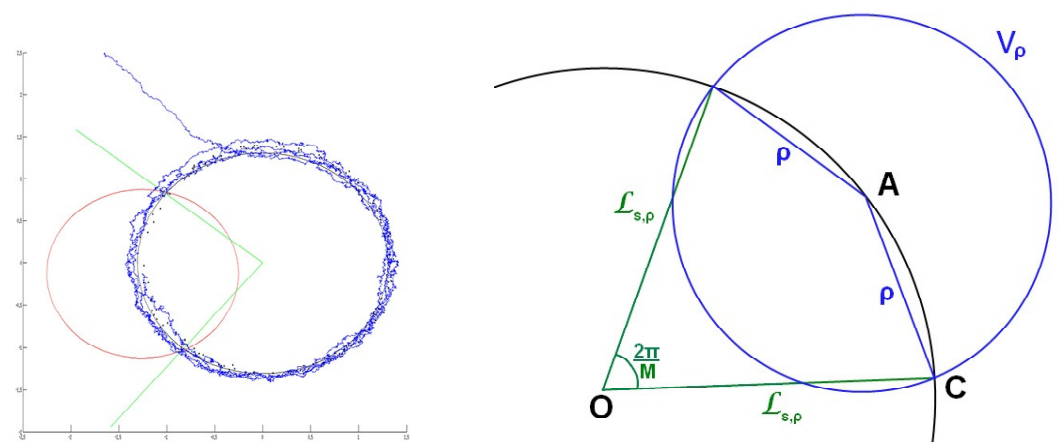

Fig. 1: Left: An example run with $N=100$ agents, and a range $\rho=1$. In black, the theoretic limit cycle, and in blue the positions through time of one agent.

Right: Geometric explanation of the computation of $\mathcal{L}_{s, \rho}$ : In the stationary regime, each agent has in average $\frac{N}{M}$ agent in her neighborhood. As the agents are uniformly distributed on the limit cycle, the number of neighbors is directly correlated with the arc length contained within the neighborhood of each agent. Thus each agent's range exactly encompasses an arc of $\frac{2 \pi}{M}$, and the rule of cosine in the triangle OAC leads to the result of Eq.(11).

\section{Swarm Soft Controlling - Harmonic oscillator Hamiltonian}

Now we focus on cylindrically symmetric configurations involving the Hamiltonian Eq.(10), and for these cases we generalize Eq.(1) by allowing the agents to self-adapt their circulation velocities. This can be achieved by replacing the nominal matrix $\mathcal{A}_{k}(t)$ in Eq.(2) by $\mathbb{A}_{k}$ :

$$
\mathbb{A}_{k}=\left(\begin{array}{cc}
\frac{N_{k, \rho}(t)}{N}-\frac{1}{M} & \omega_{k}(t) \frac{N_{k, \rho}(t)}{N} \\
-\omega_{k}(t) \frac{N_{k, \rho}(t)}{N} & \frac{N_{k, \rho}(t)}{N}-\frac{1}{M}
\end{array}\right),
$$


where the now adaptive angular velocity $\omega_{k}(t)$ of agent $a_{k}$ obeys to the relaxation dynamics:

$$
\left\{\begin{array}{l}
\dot{\omega}_{k}(t)=\gamma_{1} \cdot\left[\langle\omega\rangle_{k, \rho}(t)-\omega_{k}(t)\right]+\gamma_{2} \cdot\left[\Omega-\omega_{k}(t)\right], \quad \omega_{k}(0)=\omega_{0, k}, \\
\langle\omega\rangle_{k, \rho}(t):=\frac{1}{N_{k, \rho}(t)} \sum_{j \in V_{k, \rho}(t)} \omega_{j}(t),
\end{array}\right.
$$

with $\gamma_{i}>0(i=1,2)$.

Remark. The set of ODE's in Eq.(13) describes an autonomous, systematically dissipative linear dynamical system. Accordingly, the unique resulting attractor is here given by $\lim _{t \rightarrow \infty} \omega_{k}(t)=\Omega, \quad \forall k$.

\subsection{Inhomogeneous swarm - Shill soft controlling mode}

Let us now introduce an externally controllable agent, to be called the shill (SH) agent, into the swarm. Agent $\mathrm{SH}$ is, without loss of generality, taken as $a_{1}$ and we assume that we can fix $\omega_{1}(t) \equiv \omega_{s}$. Regarding the radial dynamics, we assume that $\mathrm{SH}$ behaves as an ordinary agent. In presence of $\mathrm{SH}$, the swarm is heterogeneous, thus precluding analytical approaches for general parameter ranges. Nevertheless, for limiting regimes, we will now see that our dynamics still lend itself to an approximate analytical treatment.

We assume in Eq.(1) that the signal to noise ratio (SNR) $\left(\Lambda / \sigma^{2}\right)<<1$. In absence of $\mathrm{SH}$, the stationary regime of the homogeneous swarm uniformly distributes the agents along the arcs of a narrow annular ring with radius $\mathcal{L}_{s, \rho}$. The agents circulate with a common angular velocity $\omega_{k} \equiv \Omega$. Let us now introduce $\mathrm{SH}$ into the swarm.

We shall consider the cases where $\omega_{s}>\Omega$ (the other possibilities can be discussed along the same lines). Taking into account the presence of SH in the swarm, our goal is to first show that for $\langle\omega\rangle_{k, \rho}(t)$ in Eq.(13), we can approximately write:

$$
\langle\omega\rangle_{k, \rho}(t) \simeq \begin{cases}\frac{\omega_{s}+\left(\frac{N}{M}-1\right) \omega_{k}(t)}{N / M} & \text { when } \quad S H \in V_{k, \rho}(t), \\ \omega_{k}(t) & \text { otherwise. }\end{cases}
$$

To heuristically derive Eq.(14), we assume that at initial time $t=-\infty$, we have an homogeneous population involving $N$ agents. At time $t=0^{-}$, this homogeneous swarm has reached its stationary regime, i.e. the agents are confined in a ring close to the cycle $\mathcal{L}_{s, \rho}$ and their stationary circulation has the common angular velocity $\omega_{k} \equiv \Omega$. For times $t \geq 0^{+}$, we switch on SH's action by imposing $\omega_{1}(t) \equiv \omega_{s}>\Omega$. Via mutual interactions, SH is able to enhance the $a_{k}$ 's angular velocities for $k=2, \ldots, N$. Therefore, besides the intrinsic dissipation mechanism given in Eqs.(1) together with Eqs.(12) and (13), the $a_{k}$ 's $(k=2, \ldots, N)$ do effectively scavenge rotational energy from $\mathrm{SH}$ and ultimately an energy balance will be reached.

For large populations $N$, we assume SH's influence to be quasi-adiabatic. That is to say, we assume that the $a_{k}$ 's distribution on the limit cycle $\mathcal{L}_{s, \rho}$ remains 
essentially unaltered. Since we choose SH's angular speed $\omega_{s} \equiv \omega_{1}>\Omega$ and because $\mathrm{SH}$ itself circulates with high probability inside the thin annulus $\mathcal{L}_{s, \rho}$, SH periodically crosses the $V_{k, \rho}(t)$ for $k=2,3, \ldots, N$. During the SH's transit time inside $V_{k, \rho}(t)$, interaction with SH enhances $\omega_{k}$. Conversely, when SH lies outside $V_{k, \rho}(t)$, then $\omega_{k}$ freely relaxes towards $\Omega$. To be consistent with the adiabatic energy exchange assumption, the SH's influence on $\omega_{k}$ during one $V_{k, \rho}(t)$ over-crossing has to remain small. At a given time $t$, and for a tagged $a_{k}(k=2,3, \ldots, N)$, two configurations are alternatively realized depending on whether SH belongs to $V_{k, \rho}(t)$ or not:

i) $\mathrm{SH} \notin V_{k, \rho}(t)$ : only regular neighbors surround $a_{k}$. Their individual speed very slightly differs from $\omega_{k}(t)$. These differences are due to SH leaving the neighboring $a_{k}$ 's $V_{k, \rho}(t)$ at different successive times. Some of the neighbors of $a_{k}$ will have an angular velocity slightly higher than $\omega_{k}(t)$ (the rapids), while others will have a slightly lower angular velocities (i.e. the slows). SH's constant rotation, always leaves a wake of agents with a slight angular velocity enhancement in SH's trail. Constant rotation implies that the rapids and slows are approximatively spatially distributed symmetrically with respect to $a_{k}$ and close to the cycle $\mathcal{L}_{s, \rho}$. As the $a_{k}$ 's neighbors present in $V_{k, \rho}(t)$ are approximately uniformly distributed on the cycle $\mathcal{L}_{s, \rho}$, on average the numbers of rapids and slows are identical. Hence, the average speed of the agents in $V_{k, \rho}(t)$ is approximately $\omega_{k}(t)$ (i.e. the rapids approximately compensate for the slows).

ii) $\mathrm{SH} \in V_{k, \rho}(t)$ : here we invoke the fact that $a_{k}$ has in average $\frac{N}{M}$ neighbors. Using i) for the $\frac{N}{M}-1$ regular neighbors of $a_{k}$ and taking into account SH's presence, the weighted average of the angular velocities leads to the first line in Eq.(14).

Using Eq.(14) into Eq.(13) enables to write:

$$
\dot{\omega}_{k}(t)=\left\{\begin{array}{l}
\left(\gamma_{1} \frac{M}{N}+\gamma_{2}\right) \cdot[\overbrace{\frac{\gamma_{1} \frac{M}{N} \omega_{s}+\gamma_{2} \Omega}{\gamma_{1} \frac{M}{N}+\gamma_{2}}}^{\nu_{1}}-\omega_{k}(t)], \\
\gamma_{2} \cdot[\underbrace{\Omega}_{\nu_{2}}-\omega_{k}(t)] .
\end{array}\right.
$$

For an ultra-fast relaxation regime $\gamma_{k} \rightarrow \infty(k=1,2)$, Eq.(15) implies:

$$
\omega_{k}(t) \simeq \begin{cases}\nu_{1} & \text { when SH in } V_{k, \rho}(t), \\ \nu_{2} & \text { otherwise. }\end{cases}
$$

Now, we can calculate the time spend into the alternative states of Eq.(16). To this aim consider Figure 2, where we sketch the approximative resulting behavior for an arbitrary agent $k$ together with $S H$.

In view of Figure 2, we now can write:

$$
\left\{\begin{array}{lll}
\frac{2 \pi}{M}+T_{1} \cdot \nu_{1}=T_{1} \cdot \omega_{s} & \Rightarrow & T_{1}=\frac{2 \pi}{M\left(\omega_{s}-\nu_{1}\right)}, \\
\frac{p i}{M}+T_{2} \cdot \omega_{s}=T_{2} \cdot \nu_{2}+\frac{2 M-1}{M} \pi & \Rightarrow & T_{2}=\frac{(2 M-2) \pi}{M\left(\omega_{s}-\nu_{2}\right)} .
\end{array}\right.
$$




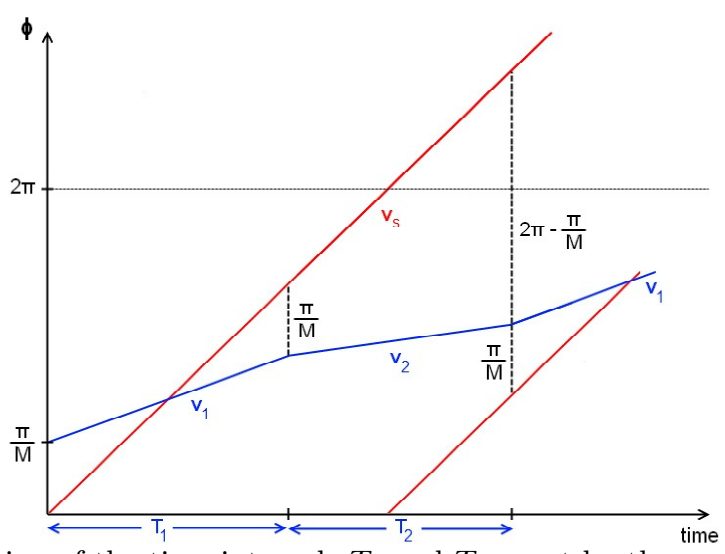

Fig. 2: Computation of the time intervals $T_{1}$ and $T_{2}$ spent by the agents in each of the states of Eq.(16). The state 1 starts when SH enters the agent's range (that is when their phase differs by $\frac{\pi}{M}$ in one direction), and lasts until they differ by $\frac{\pi}{M}$ in the other direction. Then, the time interval $T_{2}$ lasts until $\mathrm{SH}$ re-enters the agent's range, that is when their phases differ of $\frac{2 M-1}{M} \pi$.

Hence, on a single time period $T=T_{1}+T_{2}$, the resulting weighted average angular velocity yields:

$$
\omega_{\text {ave }}=\frac{T_{1} \cdot \nu_{1}+T_{2} \cdot \nu_{2}}{T_{1}+T_{2}}=\underbrace{\frac{\gamma_{2} N}{\gamma_{1}+\gamma_{2} N}}_{\alpha\left(N, \gamma_{1}, \gamma_{2}\right)} \cdot \Omega+\underbrace{\frac{\gamma_{1}}{\gamma_{1}+\gamma_{2} N}}_{\beta\left(N, \gamma_{1}, \gamma_{2}\right)} \cdot \omega_{s} .
$$

Note that $\alpha\left(N, \gamma_{1}, \gamma_{2}\right)+\beta\left(N, \gamma_{1}, \gamma_{2}\right)=1$ and $\left[\alpha\left(N, \gamma_{1}, \gamma_{2}\right) / \beta\left(N, \gamma_{1}, \gamma_{2}\right)\right]=N\left(\gamma_{2} / \gamma_{1}\right)$. Hence, we consistently observe that $\lim _{N \rightarrow \infty} \alpha\left(N, \gamma_{1}, \gamma_{2}\right)=1$, showing that the SH's relative influence is reduced with the population size $N$.

Figure 3 shows numerical results for a swarm of $N=2000$ agents for time $t \in[0 ; 100]$ with $M=4$. Input parameters are

$$
\left.\begin{array}{lll}
\sigma=0.5 & \rho=1 & \Omega=-0.2 \\
\gamma_{1}=40 & \gamma_{2}=1 & \omega_{s}=31.4
\end{array}\right\} \Rightarrow \omega_{\text {ave }}=0.4 .
$$

Note that some agents end up in the vicinity of the origin. These agents will asymptotically with time converge to the limit cycle $\mathcal{L}_{s \rho}$, and to the theoretic consensual angular speed $\omega_{\text {ave }}$, but that can not be seen in the small time window of these simulations. Despite these agents, the average values for the radius and the angular speed of the swarm exactly match the theoretical values.

\section{Conclusion}

The intrinsic swarm's heterogeneity due to the presence of a shill fellow into an otherwise homogeneous swarm agents offers, in general, little hope for analytically deriving the dynamical features of the global population. However for the present mixed canonical-dissipative dynamics with agent-dependent angular speed, we are able to show how reliable analytical approximations can be 

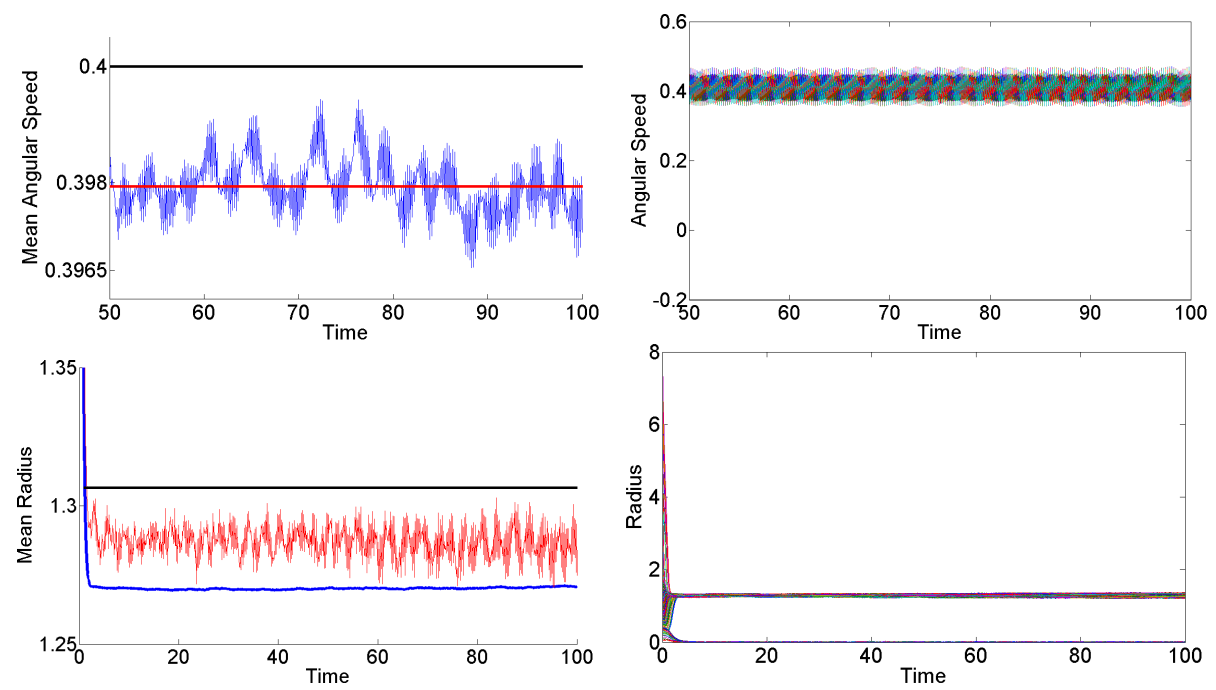

Fig. 3: Top left: In blue, the average angular speed of the regular agents, with in red its average over the period $t \in[50 ; 100]$. In black, the expected value $\omega_{\text {ave }}=0.4$. right: Individual angular speeds of each agent as a function of time. Bottom left: In blue the average radius of the regular agents, and in red the radius of SH. In black, the expected value $\mathcal{L}_{s, \rho} \simeq 1$.31. right: Individual radii of each agent as a function of time.

derived. Being able to quantitatively appreciate the shills presence, it then offers the possibility to select the optimal shills characteristics. Our analytical approach is fully corroborated by a set of numerical simulations involving large populations of magnitude order of $10^{3}$ individuals.

\section{References}

1. G. Sartoretti and Hongler M.-O. Soft control of swarms - analytical approach. In Proceedings - International Conference on Agents and Artificial Intelligence 2013.

2. J. Han Wang X. and H. Han. Special agents can promote cooperation in the population. PLoS ONE 12, vol. 6, 2011.

3. L. Wang and L. Guo. Robust consensus and soft control of multi-agent systems with noises. Journal of Systems Science and Complexity 3, vol. 21, 406-415, 2008.

4. Deneubourg J. L. H. Bleuler Gribovskiy A., Halloy J. and F. Mondada. Towards mixed societies of chickens and robots. IEEE/RSJ 2010 International Conference on Intelligent Robots and Systems, Conference Proceedings pp. 4722-4728, 2010.

5. G. Caprari C. Rivaullt M. Asadpour J. M. Ame C. Detrain N. Correl A. Martinoli J. Halloy, G. Sempo and F. Mondada. Social integration of robots into groups of cockroaches to control self-organized choices. Science, 318:1155, $200 \%$.

6. Dyer J. R. G. Faria J. J. and J. Krause. A novel method for investigating the collective behaviour of fish: Introducing 'robofish'. Behavioral Ecology and Sociobiology 8, vol. 64, 1211-1218, 2010.

7. G. Sartoretti and Hongler M.-O. Self-organized mixed canonical-dissipative dynamics for brownian planar agents. Cybernetics and Physics, In Press. 